\title{
Variação temporal do fitọplânçton em um rio tropical pré-amazônico (Rio Pindaré, Maranhão, Brasil).
}

\author{
Temporal variation of phytoplankton in a pre-amazonian tropical river \\ (Pindaré River, Maranhão, Brazil)
}

\author{
Eduardo Henrique Costa Rodrigues ${ }^{* 1}$, Larissa Nascimento Barreto ${ }^{2}$, \\ Maria Marlúcia Ferreira-Correia ${ }^{3}$ e Maria Raimunda Chagas Silva ${ }^{4}$ \\ 1,4 Professores do Curso de Engenharia Ambiental, Campus Renascença , \\ Universidade do Ceuma, São Luis, Maranhão, Brasil \\ ${ }^{2,3}$ Professores do Departamento de Oceanografia e Limnologia, \\ Universidade do Ceuma, São Luis, Maranhão, Brasil
}

\begin{abstract}
Resumo
O presente estudo objetivou analisar a variação temporal do fitoplâncton do médio curso do Pindaré. Foram coletadas amostras de água em seis pontos distintos ao longo do médio curso do rio Pindaré durante os picos dos períodos chuvoso e de estiagem. O fitoplâncton foi amostrado com rede de plâncton de $20 \mu \mathrm{m}$ de malha e garrafa de Von Dorn; as amostras foram preservadas em formalina $4 \%$ e lugol respectivamente. O rio Pindaré apresenta elevada biodiversidade de microalgas planctônicas estando estas representadas por um total de 98 espécies distribuídas em seis classes. A classe Bacillariophyceae se mostrou a mais bem representada enquanto as Cyanophyceae foram as menos diversificadas. O fitoplâncton apresentou maiores valores de densidade durante o período chuvoso e menor durante a estiagem. A diversidade e a equitabilidade foram altas durante todo o período de estudo. A composição da comunidade fitoplanctônica sofreu, no ano de estudo, uma nítida variação sazonal.
\end{abstract}

Palavras-chave: Fitoplâncton. Variação Temporal. Rio Pindaré. Pré-Amazônia

\begin{abstract}
The purpose of the present study was to analyze the temporal variation of phytoplankton in th middle course of Pindare river. Water samples were collected in six different spots along the Pindaré river middle course during the peaks of rainy and dry seasons. The phytoplankton was sampled with a plankton net of a 20 um mesh and Von Dorn bottle. The samples were preserved in formalin $4 \%$ and lugol respectively. The Pindaré River contains high biodiversity of plankton microalgae and it is represented by a total of 98 species distributed in six classes. The Bacillariophyceae class is the best represented whereas the Cyanophyceae class is the least diversified. The phytoplankton showed higher density values during rainy season and lower values during dry season. The diversity and equitability were high throughout the study period. The composition of the phytoplankton community suffered a clear seasonal variation, during the year of study.
\end{abstract}

Keywords: Phytoplankton. Temporal Variation. Pindaré River. Pre-Amazon 


\section{Introdução}

$\mathrm{O}$ fitoplâncton pode ser definido como um aglomerado de organismos fotossintetizantes em que a capacidade de locomoção na coluna d'água é praticamente inexistente ou quando esta ocorre, é de modo deficiente. A comunidade fitoplanctônica vive em suspensão durante todo o seu ciclo de vida e a fase vegetativa, na coluna d'água (ESTEVES, 2011). Esses organismos são encontrados em todos os lagos, rios de baixa correnteza, estuários e oceanos. Os organismos que formam o fitoplâncton são provavelmente os organismos fotossintetizantes mais comuns no mundo (HORNE, 1994).

A variação das comunidades fitoplanctônicas ao longo do tempo é um dos conhecimentos mais antigos em limnologia (ESTEVES, 2011). Isso porque o fitoplâncton está sujeito a fortes influências sazonais ou temporais. Em zonas temperadas e polares existe um grande contraste entre a primavera, o verão, o outono e o inverno. Na região intertropical a variação do fitoplâncton é influenciada pela diferença entre as épocas de chuvas e a estação de estiagem. As algas respondem a esses constantes rearranjos nas características físicas e químicas em seu ambiente com flutuações populacionais (florações) características (HORNE, 1994). Assim, é fácil entender que a influência das estações do ano sobre o fitoplâncton em regiões não tropicais do globo apresenta pouca relevância. Em regiões tropicais a periodicidade do fitoplâncton não é uniforme e existem poucas evidências para a ocorrência de flutuações ligadas às estações do ano (flutuações sazonais). Desta maneira, para o fitoplâncton de regiões tropicais seria mais aconselhado falar-se em variação temporal (ESTEVES, 2011).

A importância do estudo do fitoplâncton dá-se pelo fato deste, juntamente com outros organismos autotróficos, constituir a base da teia trófica em ambientes aquáticos. Esses organismos são ainda responsáveis pela produtividade primária desses ambientes, atuando assim como "(re)cicladores" da matéria em ecossistemas aquáticos. Além disso, a comunidade fitoplanctônica é amplamente utilizada em estudos de biomonitoramento de corpos hídricos (CARVALHO, 2003) em função da sua capacidade ecológica em responder rapidamente a qualquer estímulo externo, como por exemplo, aos processos de poluição e contaminação do meio aquático. Estes organismos são muito utilizados como bioindicadores da qualidade ambiental (AQUINO et al., 2011; LOPES, 2007; PEREIRA, 2007).

No Brasil e no mundo diversos estudos têm sido realizados para examinar a ecologia e variação temporal da assembleia fitoplanctônica, e como se dá sua distribuição espaço-temporal em ambientes aquáticos, como rios e lagos.
No estado do Maranhão a variação sazonal/temporal e a ecologia do fitoplâncton de águas interiores foi estudada por muitos pesquisadores: dentre eles encontram-se trabalhos de Barbieri et al. (1989), Araujo et al. (1998), Pompeo et al. (1998), Moschini-Carlos; Pompeo (2001), Dellamano-Oliveira; Senna e Taniguchi (2003), Almeida et al. (2005), Nogueira et al. (2005), MoschiniCarlos et al. (2008).

Este trabalho teve como objetivo realizar um estudo sobre a variação temporal da comunidade fitoplanctônica do rio Pindaré analisando-se as modificações na densidade, frequência de ocorrência, diversidade e na abundância entre outros parâmetros.

\section{Material e Método}

\section{1 Área de estudo}

A Pré-Amazônia maranhense representa uma área de transição entre duas regiões brasileiras com características distintas e bem marcantes:o Nordeste do Brasil, tipicamente seco e com precipitações pouco volumosas, e o Norte, considerado uma região de elevada umidade e precipitações frequentes e bem distribuídas. Essa região não apresenta o marcante déficit hídrico característico dos demais estados nordestinos (POMPÊO; MOSCHINI-CARLOS; SILVA-FILHO, 2002).

O rio Pindaré (Fig. 01) é, segundo Pompêo, MoschiniCarlos e Silva-Filho (2002), um rio tropical, localizado na Pré-Amazônia Maranhense, estando sua bacia hidrográfica sujeita aos regimes climáticos característicos das regiões norte (elevada pluviosidade) e nordeste (períodos prolongados de estiagem). Este rio representa o principal afluente do rio Mearim.

$\mathrm{O}$ rio nasce nas elevações que formam o divisor de águas das bacias hidrográficas dos rios Mearim e Tocantins, possuindo um percurso total de aproximadamente $686 \mathrm{~km}$ (BACIAS DO NORDESTE, 2000).

A bacia hidrográfica do rio Pindaré tem aproximadamente $44.400 \mathrm{~km}^{2}$ de área estando situada na região centro-ocidental do estado fazendo parte do bioma Pré-Amazônia do Maranhão. Nessa área a cobertura vegetal é classificada como floresta ombrófila densa, seu clima é do tipo Úmido (B1) e (B2), com duas estações bem definidas, uma seca e uma chuvosa, ambas com duração media de seis meses cada, a temperatura média anual é de $26^{\circ} \mathrm{C}$ e a precipitação anual média varia de 1200 - $1600 \mathrm{~mm}$; o clima é quente na estação seca e mais ameno no período de chuvas (MARANHÃO, 2002).

Destaca-se entre os demais rios maranhenses pela elevada piscosidade e pela importância à navegação local, bem como ao abastecimento publico nas áreas por onde passa. 


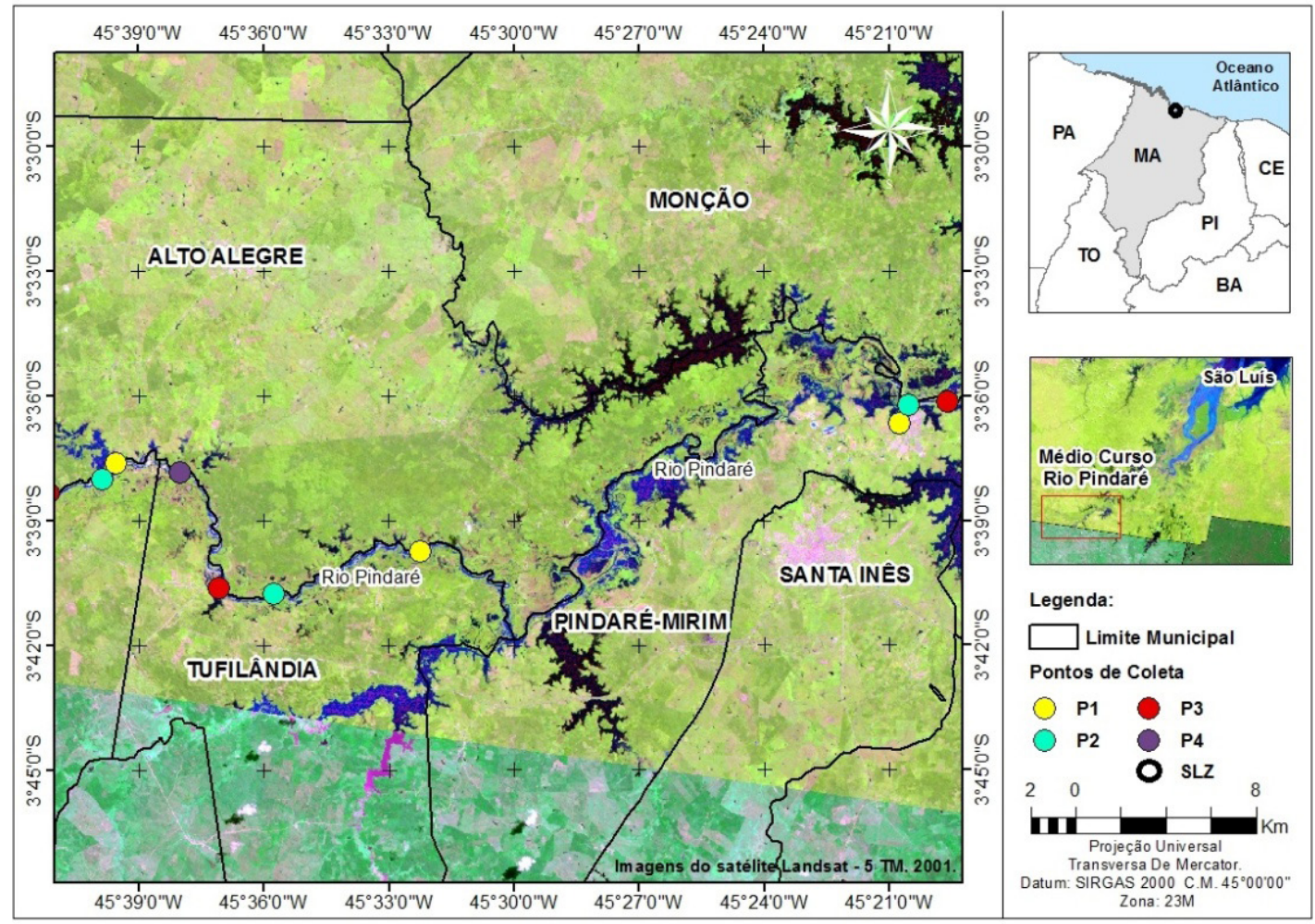

Figura 01- Mapa da área de estudo evidenciando as estações de coleta ao longo do médio curso do rio Pindaré. P1-Calixto; P2-Porto das lanchas; P3- Prainha; P4- Poção do Geraldo e P5- Barraca Feliz P6-Plantação de Arroz. SLZ - São Luis (Capital do estado) Fonte: Dados da pesquisa

\subsection{Coleta de dados}

Os aspectos ecológicos do fitoplâncton foram determinados através de amostras coletadas em seis pontos fixos distribuídos ao longo do médio curso do rio Pindaré. As coletas ocorreram durante o período chuvoso (maio/2011) e o período de estiagem (outubro/2011) da região.

As estações de coleta foram distribuídas entre as cidades de Tufilândia (S 03 40, 863' W $\left.043^{\circ} 36,557^{\prime}\right)$, com Pontos P1 e P2, P3 e P4 e Alto Alegre do Pindaré (S $\left.03^{\circ} 37,617^{\prime} \mathrm{W} 045^{\circ} 39,533^{\prime}\right)$, com Pontos P5 e P6.

Para a análise qualitativa, referente às identificações das espécies do fitoplâncton e determinação da riqueza especificas, foram realizadas coletas com rede de malha de $20 \mu \mathrm{m}$ em arrastos horizontais na superfície da água em intervalos de tempo de aproximadamente cinco minutos cada. As amostras foram fixadas com formol, $4 \%$ armazenadas em frascos plásticos com capacidade de $250 \mathrm{~mL}$ cada. O material coletado nessa etapa também foi destinado à construção da matriz de presença e ausência objetivando o estudo de similaridade entre os pontos amostrais estudados.

Para análise quantitativa (densidade celular) do fitoplâncton, foram realizadas coletas com garrafa coletora do tipo Van Dorn em mergulhos na coluna d'água em meio à zona eufótica. As amostras obtidas com essa técnica foram fixadas com lugol. A densidade fitoplanc- tônica foi determinada através de contagem de campos iluminados (total de 100) em invertoscópio segundo metodologia de Utermöhl (1958).

A identificação dos táxons foi baseada em bibliografia especializada seguindo recomendações de Bicudo e Menezes (2006), Bicudo e Bicudo (1970), Dellamano-Oliveira et al. (2008), Laux e Torgan (2011) e Oliveira, Torgan e Rodrigues (2002). A identificação das diatomáceas foi baseada nas características morfológicas e morfométricas. Foram produzidas lâminas permanentes com material oxidado segundo metodologia Müller-Melchers e Ferrando (1956). As leituras ocorreram em microscópios ópticos com aumento de 400x. Foi observado o máximo de indivíduos possíveis de uma mesma espécie para estudo morfológico mais detalhado. Os táxons identificados foram comparados aos registros pré-existente (fotografias e descrição taxonômicas) disponibilizados no laboratório de Fitoplâncton do Departamento de Oceanografia e Limnologia da Universidade Federal do Maranhão - DEOLI.

A frequência de ocorrência das espécies foi determinada segundo metodologia de Mateucci; Colma (1982). Os cálculos da diversidade e equitabilidade foram realizados, utilizando-se o Índice de Shannon. A análise de diversidade foi realizada através do programa PAST (HAMMER, 2001). Para verificar se há diferença na abundância por espécie entre os diferentes pontos nos períodos de estiagem e chuvoso foi aplicado o teste de 
similaridade unifatorial ANOSIM, realizado no programa PAST (HAMMER, 2001).

A frequência, a diversidade, a equitabilidade, a abundância e a similaridade foram obtidas através de análise do material proveniente da amostragem livre com auxilio da garrafa de Von Dorn. A similaridade dos pontos amostrais foi realizada através da análise de agrupamento de CLUSTER utilizando-se o programa PRIMER (versão 5.0). Para esta análise, os dados utilizados foram baseados na presença e/ou ausência das espécies nos pontos de coleta através do material identificado da rede de fitoplâncton.

Todas as análises foram realizadas separadamente por estações considerando-se que a ocorrência de muitas espécies muda de um período para outro.

\section{Resultados}

A comunidade fitoplanctônica do rio Pindaré esteve representada por uma considerável diversidade de organismos. O fitoplâncton no médio curso do rio Pindaré, apresentou 98 táxons distribuídos em seis classes taxonômicas: Bacillariophyceae (34\%), Cyanophyceae $(8 \%)$, Chlamydophyceae (4\%), Chlorophyceae, $(26 \%)$, Euglenophyceae (15\%), Zygnemaphyceae (13\%) (Fig. 2). Foram registradas 88 espécies no fitoplâncton no período chuvoso e apenas 63 durante a estiagem na região.

A classe Bacillariophyceae foi predominante durante os períodos de estudo, na estiagem e na época de chuvas. A classe Chlorophyceae foi a segunda em termos de riqueza de espécies, apresentando uma boa participação em ambos os períodos.

As diatomáceas formaram o grupo com maior ri- queza especifica apresentando 13 gêneros e 33 espécies, seguidas pela classe Chlorophyceae com 14 gêneros e 26 espécies, a classe Euglenophyceae registrou 04 gêneros e 15 espécies. Os organismos pertencentes à classe Chlamidophyceae apresentaram menor riqueza durante todo período de estudo, registrando apenas 03 gêneros e 04 espécies (Fig. 03).

Tanto a distribuição das classes quanto a riqueza foram analisadas através do material amostrado com a rede de fitoplâncton.

A densidade total do fitoplâncton foi um parâmetro que variou temporalmente, oscilando entre $244 \times 10^{3}$ cel.L $\mathrm{L}^{-1}$ a $1348 \times 10^{3}$ cel. $L^{-1}$, apresentando valores mais elevados durante o período de estiagem (Fig. 4).

Espacialmente a densidade do fitoplâncton decresceu no sentido Tufilândia a Alto Alegre durante os dois períodos de estudo (chuvoso e estiagem) (Fig. 4). A classe Euglenophyceae foi a maior contribuinte para a densidade durante o período chuvoso com um total estimado de indivíduos da ordem de $1400 \times 10^{3}$ cel.L-1, levando-se em consideração o somatório da densidade entre os pontos analisados.

Durante a estiagem, as classes que registraram maiores valores de densidade foram Chlorophyceae $1650 \times 10^{3}$ cel.L ${ }^{-1}$ e Bacillariophyceae $1606 \times 10^{3}$ cel.L $L^{-1}$ respectivamente (Fig. 4).

As espécies de diatomáceas Eunotia didyma Grunow e Ulnaria ulna (Nitzsch) P. Compère, os fitoflagelados Euglena proxima P.A.Dangeard e Trachelomonas volvocina (Ehrenberg) Ehrenberg foram consideradas as mais frequentes em ambos os períodos estudados, durante a seca (estiagem) e época de chuvas. Além dessas espécies um gênero da família Zygnemaphyceae, o Closterium leibleinii Kützing ex Ralfs, foi considerado muito frequente.

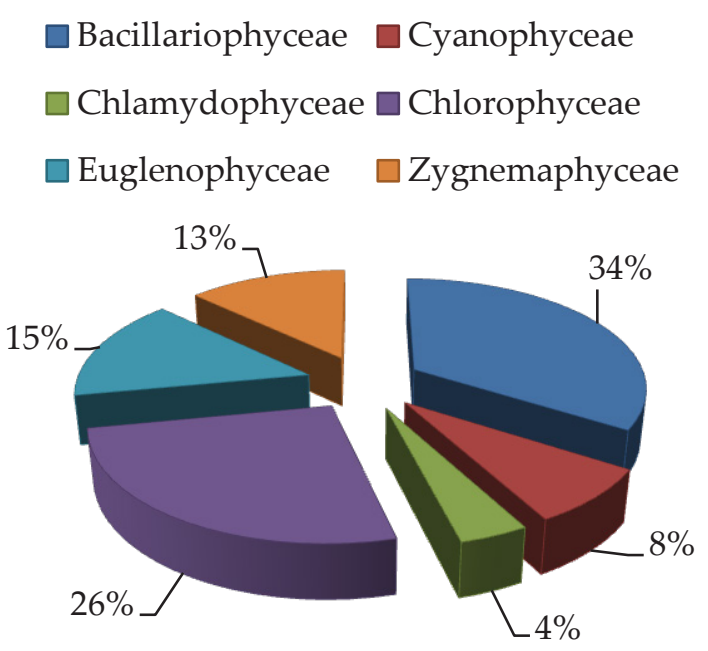

Figura 02 - Distribuição percentual das diferentes classes da comunidade fitoplanctônica no médio curso do rio Pindaré durante os períodos (estiagem e chuvoso) 


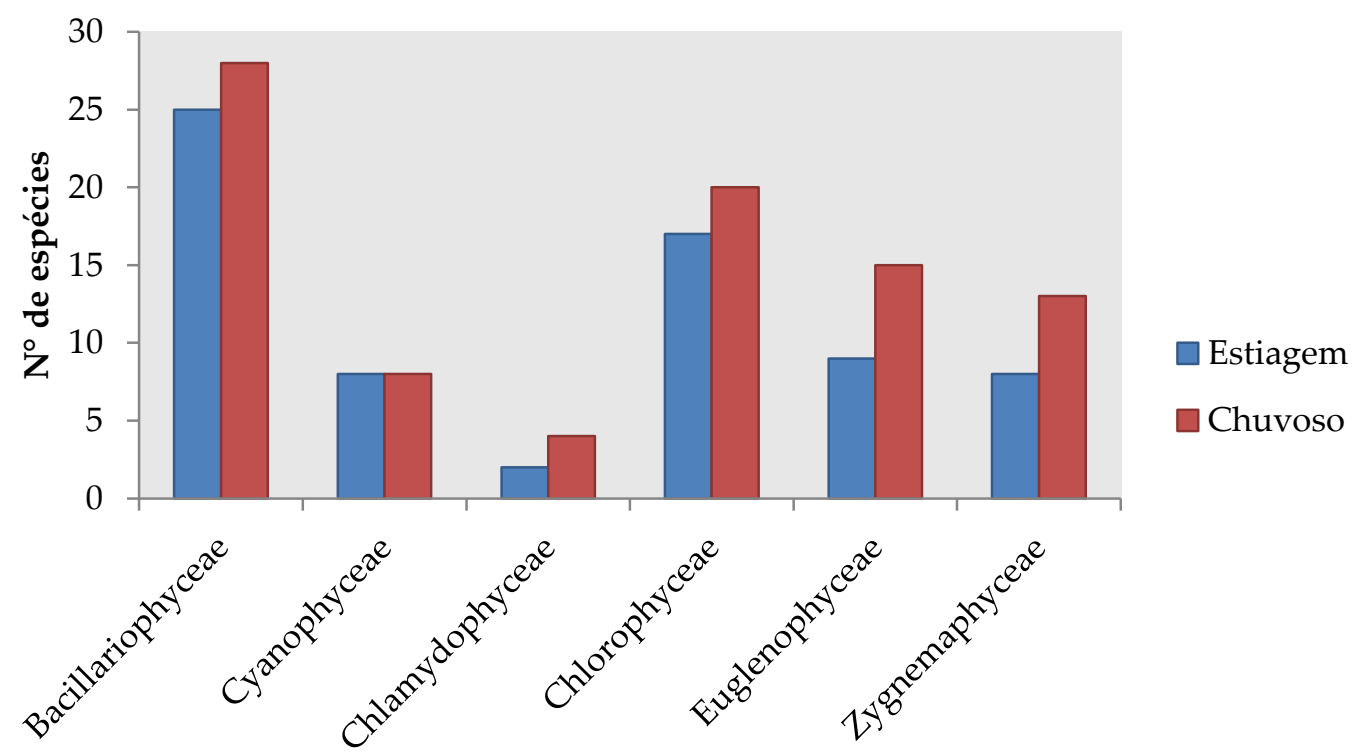

Figura 03 - Variação total do número de espécies (riqueza) por classe taxonômica durante o durante os períodos (estiagem e chuvoso)

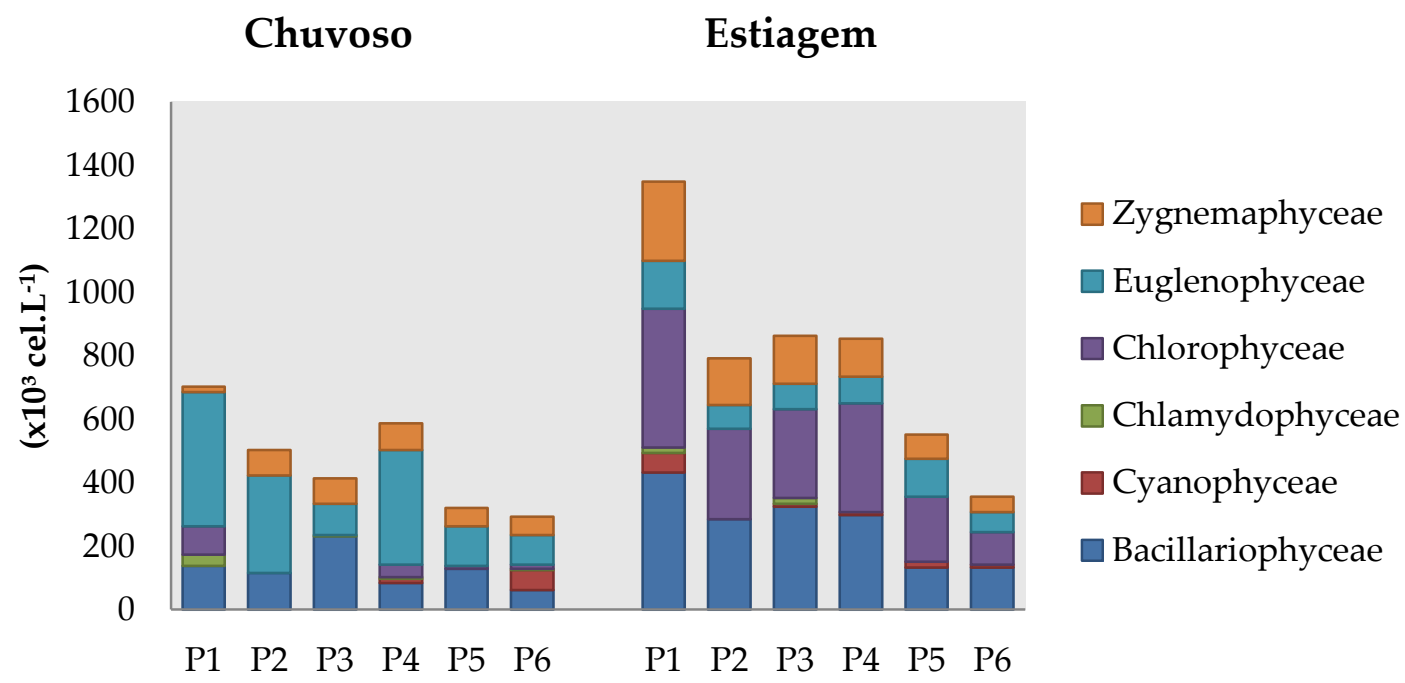

Figura 4 -Variação da densidade por classe nos pontos de amostragem no médio curso do rio Pindaré. P1-Calixto; P2-Porto das lanchas; P3- Prainha; P4- Poção do Geraldo P5- Barraca Feliz P6-Plantação de Arroz, durante os períodos (estiagem e chuvoso)

A família Bacillariophyceae apresentou maior frequência de ocorrência, registrando $29 \%$ de espécies frequentes durante o período chuvoso, durante a estiagem esse valor caiu para $21 \%$.

Vinte e cinco por cento das espécies que constituem a família Chlorophyceae foram consideradas frequentes durante o período chuvoso. Durante a época de estiagem, essa classe registrou apenas uma espécie muito frequente, a Ankistrodesmus falcatus (Corda) Ralfs

Independentemente do período em que foram identificadas neste estudo, todas as cianobactérias (classe
Cyanophyceae) foram consideradas esporádicas ou pouco frequentes não apresentando frequência de ocorrência inferior a $33,3 \%$.

Do total de espécies identificadas, 12 (doze) foram exclusivas do período de estiagem. Deste total, aproximadamente $58 \%$ eram algas pertencentes à classe Chlorophyceae. Por outro lado, foram registradas 28 (vinte e oito) espécies exclusivas do período chuvoso sendo que deste total, $25 \%$ eram representantes da classe Euglenophyceae.

Não foram observadas espécies que ofereçam risco à 
saúde humana ou à qualidade do meio ambiente através da produção de toxinas. Contudo, ao longo do rio, foram identificadas grandes áreas desmatadas e com forte ação antrópica, podendo oferecer risco à qualidade da água, futuramente, pois sem a cobertura vegetal natural um maior aporte de material escoa para o rio, contribuindo assim para o assoreamento do canal.

A distribuição relativa da abundância das classes apresentou diferenças em relação aos pontos estudados e à época de amostragem. Durante a estiagem as diatomáceas foram os organismos com maior abundância relativa no fitoplâncton. Nesse mesmo período, a abundância

Tabela 3 - Relação das espécies que compõem a comunidade fitoplanctônica identificada durante os períodos (estiagem e chuvoso) de estudo no rio Pindaré

\begin{tabular}{|c|c|c|}
\hline \multicolumn{3}{|c|}{ Bacillariophyceae } \\
\hline Actinastrum hantzschii Lagerheim & Eunotia muscicola Krasske & Pinnularia acrosphaeria W.Smith \\
\hline Aulacoseira granulata (Ehrenberg) & Eunotia pectinalis var. ventricosa & Pinnularia viridis (Nitzsch) \\
\hline Simonsen & (Ehrenberg) Grunow & Ehrenberg \\
\hline Encyonema sp. & Eunotia ventriosa R.M.Patrick & $\begin{array}{l}\text { Stenopterobia curvula (W.Smith) } \\
\text { Krammer }\end{array}$ \\
\hline Eunotia anamaragaritae Metzeltin & E. ventriosa var. brevis (Patrick) & Surirella angusta Kützing \\
\hline \& Lange-Bertalot & D.Metzeltin \& Lange-Bertalot & \\
\hline Eunotia camelus Ehrenberg & Fragilaria capucina Desmazières & Surirella gracilis (W.Smith) Grunow \\
\hline $\begin{array}{l}\text { Eunotia flexuosa (Brébisson ex } \\
\text { Kützing) Kützing }\end{array}$ & Gomphonema augur Ehrenberg & Surirella linearis W.Smith \\
\hline Eunotia formica Ehrenberg & Gyrosigma sp.1 & $\begin{array}{l}\text { Surirella linearis var. constricta } \\
\text { Grunow }\end{array}$ \\
\hline Eunotia didyma Grunow & Gyrosigma sp.2 & Surirella robusta Ehrenberg \\
\hline $\begin{array}{l}\text { E. didyma fo. elongata (Grunow) } \\
\text { Hustedt }\end{array}$ & Melosira granulosa O.Müller & $\begin{array}{l}\text { Surirella robusta var. splendida } \\
\text { (Ehrenberg; Ehremberg) Van Heurck }\end{array}$ \\
\hline Eunotia major (W.Smith) & Melosira granulata var. angustissima & Surirella tenera W.Gregory \\
\hline Rabenhorst & Otto Müller & \\
\hline Eunotia monodon Ehrenberg & Nitzschia obscura Grunow & Ulnaria ulna (Nitzsch) P. Compère \\
\hline \multicolumn{3}{|c|}{ Cyanophyceae } \\
\hline Anabeana sp. & Microsystis sp. & Planktosphaeria gelatinosa G.M.Smith \\
\hline $\begin{array}{l}\text { Coelosphaerium chlamydocystis } \\
\text { Skuja }\end{array}$ & Lyngbya sp. & Pseudanabaena schmidlei Jaag \\
\hline $\begin{array}{l}\text { Merismopedia elegans A.Braun ex } \\
\text { Kützing }\end{array}$ & Oscillatoria sp. & \\
\hline \multicolumn{3}{|c|}{ Chlamydophyceae } \\
\hline Coelosphaerium $s p$ & Eudorina elegans Ehrenberg & Eudorina unicocca G.M.Smith \\
\hline Pandorina morum (O.F.Müller) & & \\
\hline Bory de Saint-Vincent & & \\
\hline
\end{tabular}

Ankistrodesmus falcatus (Corda)
Ralfs
Ankistrodesmus falcatus var.
tumidus (West \& West) G.S.West
Ankistrodesmus fusiformis Corda ex
Korshikov
Crucigenia fenestrata (Schmidle)
Schmidle
Coelastrum cambricum W.Archer
Coelastrum microporum Nägeli
Desmodesmus denticulatus
(Lagerheim) S.S.An, T.Friedl \&
E.Hegewald
Dimorphococcus lunatus A.Braun

Eudorina unicocca G.M.Smith

Eutetramorus sp.

Gloeocystis gigas (Kützing)

Lagerheim

Micractinium pusillum Fresenius

Nephrocytium agardhianum Nägeli

Pediastrum duplex Meyen

Pediastrum duplex var. gracile Roll

Pediastrum tetras (Ehrenberg) Ralfs

Scenedesmus acuminatus (Lagerheim) Chodat

Scenedesmus acuminatus var.

elongatus G.M.Smith
Scenedesmus acutus Meyen

Scenedesmus linearis Komárek

Scenedesmus protuberans F.E.Fritsch \& M.F.Rich

Scenedesmus quadricauda Chodat

Selenastrum gracile Reinsch

Tetraëdron quadrilobum G.M.Smith

Tetraëdron regulare Kützing

Tetraspora lacustris Lemmermann

Continua... 
Tabela 3 - Relação das espécies que compõem a comunidade fitoplanctônica identificada durante os períodos (estiagem e chuvoso) de estudo no rio Pindaré. Continuação...

\begin{tabular}{lll}
\hline & \multicolumn{1}{c}{ Euglenophyceae } & \\
\hline Euglena acus (O.F.Müller) & Phacus margaritatus Pochmann & Trachelomonas crebea Kellicott \\
Ehrenberg & & \\
Euglena oxyuris Schmarda & Phacus suecicus Lemmermann & Trachelomonas gibberosa Playfair \\
Euglena proxima P.A.Dangeard & Strombomonas ensifera (Daday) & Trachelomonas hispida (Perty) F.Stein \\
& Deflandre & \\
Euglena texta (Dujardin) Hübner & Strombomonas verrucosa (E.Daday) & Trachelomonas magdaleniana \\
& Deflandre & Deflandre \\
Phacus longicauda (Ehrenberg) & Trachelomonas armata (Ehrenberg) & Trachelomonas volvocina (Ehrenberg) \\
Dujardin & F.Stein & Ehrenberg \\
\hline & & \\
\hline Euastrum pulchellum Brébisson & Closterium kuetzingii Brébisson & Staurastrum sebaldii Reinsch \\
Closterium abruptum West & Closterium leibleinii Kützing ex Ralfs & Xanthidium sp \\
Closterium acerosum Ehrenberg ex & Closterium setaceum Ehrenberg ex & Xanthidium hastiferum W.B.Turner \\
Ralfs & Ralfs & \\
Closterium gracile Brébisson ex & Closterium sigmoideum Lagerheim \& & \\
Ralfs & Nordstedt \\
Closterium incurvum Brébisson & Cosmarium punctatum Nordstedt & \\
\hline
\end{tabular}

Quadro 01 - Densidade total $\left(\times 10^{3}\right.$ cel.L-1 $\left.^{-1}\right)$, diversidade (bits.cel $\left.{ }^{-1}\right)$ e equidade do fitoplâncton nas estações de coleta do rio Pindaré durante os períodos chuvoso e estiagem, P1-Calixto; P2-Porto das lanchas; P3- Prainha; P4Poção do Geraldo P5- Barraca Feliz P6-Plantação de Arroz, Dominância (Dom), Diversidade (Diver.), Densidade Total (Dens), Equidade (Equi.)

\begin{tabular}{|l|c|c|c|c|c|c|c|c|c|c|c|c|}
\cline { 2 - 12 } \multicolumn{1}{c|}{} & \multicolumn{9}{c|}{ Estiagem } & \multicolumn{6}{c|}{ Chuvoso } \\
\cline { 2 - 13 } \multicolumn{1}{c|}{} & P1 & P2 & P3 & P4 & P5 & P6 & P1 & P2 & P3 & P4 & P5 & P6 \\
\hline Dom & 0,12 & 0,14 & 0,12 & 0,08 & 0,10 & 0,12 & 0,06 & 0,08 & 0,07 & 0,06 & 0,07 & 0,06 \\
Dens & 703 & 503 & 445 & 574 & 310 & 244 & 1348 & 792 & 863 & 854 & 551 & 356 \\
Diver & 2,67 & 2,38 & 2,73 & 2,82 & 2,56 & 2,62 & 3,17 & 3,97 & 3,05 & 3,01 & 2,95 & 3,02 \\
Equi & 0,42 & 0,56 & 0,53 & 0,59 & 0,61 & 0,57 & 0,56 & 0,76 & 0,59 & 0,58 & 0,59 & 0,62 \\
\hline
\end{tabular}

da classe Euglenophyceae foi relativamente baixa. Foi ainda observada uma queda na abundância das algas pertencentes à classe Chlorophyceae durante a época de chuvas. Durante o período de chuvas houve um acréscimo nas porcentagens de participação da classe Euglenophyceae no fitoplâncton.

A diversidade da comunidade fitoplanctônica apresentou valores similares entre os pontos de amostragem. Durante o período estudado, todos os pontos apresentaram alta diversidade, sendo os maiores valores observados em Tufilândia durante o período de estiagem (Figura 5). A equitabilidade foi considerada alta na maioria dos pontos durante o período estudado variando entre 0,42 no período seco (estiagem) e 0,76 durante o período chuvoso.

Os pontos de coleta localizados na cidade de Tufilândia apresentaram os valores mais elevados de dominância durante o estudo, onde foram registrados valores máximos de 0,14 , durante o período chuvoso e 0,08 durante a estiagem, pois poucas espécies devem ter contribuído com um número elevado de indivíduos.

A análise de CLUSTER mostrou que, em ambas as épocas estudadas o município de Tufilândia ficou mais distante em termos de similaridade de abundância das espécies identificadas (Fig. 5).

Houve a formação de outros grupos com características mais parecidas agrupando assim os pontos mais similares entre si.

Foi possível observar um agrupamento entre os pontos de ESTV (P5) E ESTVI (P6) durante o período de estiagem. Os pontos ESTIV (P4) e ESTIII (P3) apresentaram maior similaridade entre si, o que fez com que durante o período de chuvas estes fossem agrupados.

A variabilidade temporal da abundância dos organismos entre os diferentes pontos amostrais ao longo do médio curso do rio Pindaré, apresentou diferenças significativas entre os pontos estudados durante os diferentes períodos do ano (chuva e estiagem) R: 0,483 p: 0,0024, permutações possíveis 9999. 


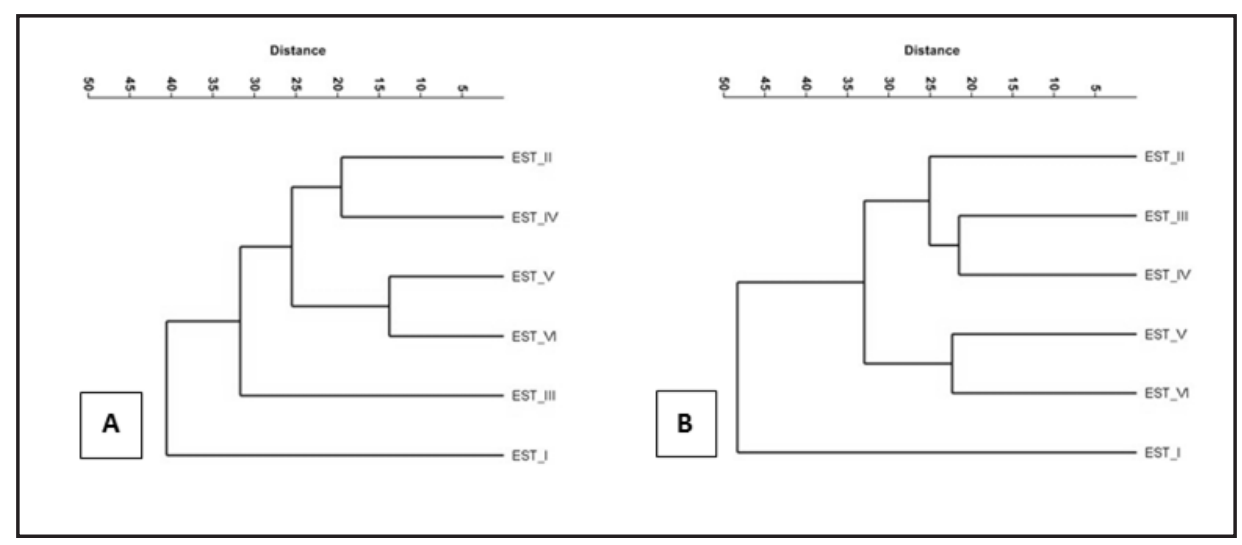

Figura 5 - Similaridade de Cluster entre os pontos estudados do rio Pindaré durante o período chuvoso (A) e seco (B) de 2011. Pontos amostrais EST_I (P1), EST_II (P2), EST_III (P3) e EST_IV (P4) (Tufilândia), EST_V (P5) e EST_VI (P6) (Alto Alegre)

\section{Discussão}

A distribuição percentual das classes taxonômicas no rio Pindaré, com predominância da classe Bacillariophyceae, é similar aos resultados observados por outros autores como Almeida et al. (2005), Araujo et al. (1998), Menezes, Bueno e Rodrigues (2013) e Pompeo et al. (1998). Pesquisa de Almeida et al. (2005) sobre a flora planctônica do Lago Cajari, durante o período de cheia, mostrou uma comunidade composta principalmente por diatomáceas e clorofíceas. O mesmo resultado foi observado por Araujo et al. (1998) ao estudar o fitoplâncton do rio Itapecuru durante o período de estiagem. A distribuição do fitoplâncton encontrada no rio Pindaré diverge da observada por Nogueira, et al. (2005) em lagos marginais do rio Turiaçu, nesses ambientes as classes que mais se destacaram foram Chlorophyceae e Zygnematophyceae, foi observada ainda uma relativa importância das cianobactérias no fitoplâncton daquela região, contando com 19 espécies registradas.

A flutuação das características hidrológicas na área de estudo levou o fitoplâncton a sofrer variações em sua ecologia, podendo ser observada nitidamente uma variação temporal em todos os parâmetros estudados. Durante a estiagem a abundância relativa das espécies que compõem a classe Chlorophyceae foi constante em todos os pontos, contudo durante o período chuvoso esta se mostrou análoga em relação aos resultados observados no período anterior, sendo considerada baixa. Essa variação deve estar ligada ao fato de no período chuvoso ocorrer grande turbulência no leito do rio e ocorrer constante suspensão de material particulado, contribuindo assim para a redução da transparência na água cooperando para a queda nos valores de densidade do fitoplâncton. Esses parâmetros são importantes na manutenção do equilíbrio ecológico do fitoplâncton. Além disso, segundo Horne (1994), os nutrientes e a luz são fatores limitantes do crescimento do fitoplâncton influenciando de maneira direta na sua ecologia. Essas modificações, no meio aquático, influenciam desde a ocorrência, através da presença ou ausência das espécies e consequentemente sua permanência na coluna d'água, até a densidade e composição especifica da comunidade planctônica.

Mudanças no ambiente provocadas pela variação das características meteorológicas, no caso a chuva e a estiagem, influenciam de forma direta nas características e variáveis bióticas das comunidades e nos ecossistemas. As características do fitoplâncton que variaram ao longo do tempo, no médio curso do rio Pindaré, podem ser resultado das modificações ocorridas nos parâmetros hidrológicos obedecendo ao gradiente temporal entre a estiagem e a época de chuvas na região, o que pode ser a resposta para as modificações sobre os valores da densidade do fitoplâncton. A densidade parece estar relacionada às flutuações do nível da água gerada pela sazonalidade do ciclo hidrológico local. Como observado, a densidade total foi maior durante período de estiagem na região, o inverso ocorreu durante o "inverno" quando a densidade foi menor.

Alguns organismos que compõem a comunidade de fitoplanctônica podem apresentar vantagens na colonização e sucessão dos ambientes onde ocorrem. No médio curso do rio Pindaré isso pode ser observado, analisando-se a variação da densidade de algumas classes, como, por exemplo, na variação da densidade das Euglenophyceae, da classe Bacillariophyceae e da Chlorophyceae. Essas classes apresentaram maiores valores durante o período de chuvas e estiagem, respectivamente. Durante o período de chuvas, quando o rio apresenta águas turvas, ricas em seston (partículas orgânicas e inorgânicas em suspensão na coluna d'água) e pouca luminosidade, houve o predomínio de euglenoides. Durante o período de estiagem, quando o rio apresenta pouca turbulência e uma transparência relativamente maior, foi observado o predomínio de algas verdes e das diatomáceas. Além disso, um acréscimo na densidade da classe Zygnemaphyceae durante a estiagem também foi observado em relação ao período chuvoso. Segundo Esteves (2011), as Euglenaceae apresentam alto grau de heterotrofia, 
vivendo em águas ricas em matéria orgânica, o que pode explicar o aumento relativo na densidade desses organismos durante período chuvas na região. Contudo, para a ecologia das algas verdes, um resultado divergente em relação a este trabalho foi observado por Granado, Henry e Tucci (2009) em estudo no rio Paranapanema. No referido trabalho, a classe Chlorophyceae contribuiu com mais de $78 \%$ da densidade total no período de cheia (chuvas na região). Segundo resultados desses autores, as diatomáceas apresentaram a segunda maior contribuição, com mais de $30 \%$ da densidade total no final da vazante e início da estiagem. Espindola (1996) sugere que vários fatores podem contribuir para as diferenças na densidade do fitoplâncton, sendo que a luz e a disponibilidade de nutrientes, ambos influenciados pelas flutuações no nível da água, parecem ser a causa mais provável para essas modificações ao longo do tempo. Ao realizar um estudo em lagos de inundação no rio Cuiabá, Loverde-Oliveira, et al. (2012) constataram que as condicionantes locais do habitat da comunidade fitoplactônica, como a transparência da água e a condutividade elétrica, foram os fatores mais importantes para a composição taxonômica e as diferentes abundâncias em cada lagoa estudada.

Algumas espécies dinamizaram sua participação no fitoplâncton, sendo registradas em apenas um período do ano ou na época de chuvas ou durante a estiagem. Como as algas refletem o padrão ambiental onde estão alocadas, elas necessitam de condições ambientais que garantam sua permanência e desenvolvimento no ambiente. Como essas condições mudam ao longo do ano com o ciclo hidrológico, algumas algas sofrem um "pulso" desaparecendo em determinado período de tempo e reaparecendo posteriormente. Espécies como Actinastrum hantzschii Lagerheim, Nitzschia obscura Grunow, Microsistis sp., Planktosphaeria gelatinosa G.M.Smith Pseudanabaena schmidlei Jaag, Desmodesmus denticulatus (Lagerheim) S.S.An, T.Friedl \& E.Hegewald, Scenedesmus acutus Meyen, Scenedesmus protuberans F.E.Fritsch \& M.F.Rich, Tetraspora lacustris Lemmermann, Phacus margaritatus Pochmann, Phacus suecicus Lemmermann, Closterium abruptum West, ocorreram somente durante a estiagem, enquanto que as espécies Eunotia anamaragaritae Metzeltin \& Lange-Bertalot, Eunotia flexuosa (Brébisson ex Kützing) Kützing, Eunotia muscicola Krasske, Eunotia pectinalis var. ventricosa (Ehrenberg) Grunow, Surirella angusta Kützing, Surirella gracilis (W.Smith) Grunow, Surirella robusta Ehrenberg, Surirella robusta var. splendida (Ehrenberg; Ehremberg) Van Heurck, Surirella tenera W.Gregory, Eudorina unicocca G.M.Smith Ankistrodesmus fusiformis Corda ex Korshikov ocorreram apenas na época de chuvas da região. Essa variação podem ser explicadas pelas variações nos parâmetros limnologicos da água do rio Pindaré, já que as diatomáceas, principalmente às pertencentes à família Eunotiaceae, são organismos comuns em ambientes ácidos (FERRARI, 2007; ALVIAL, 2008). Em estudo no médio curso do rio Pindaré, Silva
(2014) observou uma modificação nos parâmetros hidrológicos em relação aos períodos de chuvas e de estiagem na área. Dados da pesquisa mostram uma variação do $\mathrm{pH}$, entre os períodos chuvoso e de seca. Durante o período chuvoso foi possível observar valores indicando acidez da água com valores variando entre 5,6 e 5,9. Durante a estiagem a média registrada nos valores do $\mathrm{pH}$ foi 6,9. Esse fato pode explicar o "pulso" na ocorrência de algumas espécies das diatomáceas.

A riqueza específica do fitoplâncton pode estar relacionada às alterações sofridas por algumas variáveis hidrológicas do rio Pindaré, podendo os nutrientes serem os parâmetros mais preponderantes. Estudos futuros sobre as variáveis limnologicas e a relação com a flora planctônica são necessárias para confirmar essas afirmações. Segundo, Tonetta, Petrucio e Laudares-Silva (2013), alguns fatores atuam como limitantes do crescimento da comunidade fitoplanctônica, e as mudanças na concentração de nutrientes, especialmente fósforo, influenciam nas condições para o crescimento do fitoplâncton.

Segundo Melo-Magalhães (2004), o grau de complexidade da estrutura de uma comunidade é indicado pela diversidade de suas espécies, considerando o número de táxons e a equitabilidade, isto é, a distribuição dos indivíduos nos táxons. De modo geral, este trabalho mostrou que a diversidade específica (Índice de Shannon) foi considerada relativamente alta quando comparados aos trabalhos de Ahsan et al. (2012), Menezes; Bueno, Rodrigues (2013) e Offem et al. (2011). Em media esse trabalhos registram a metade da diversidade, medida pelo Índice de Shannon, observada para o rio Pindaré.

No rio Pindaré, os maiores valores da diversidade ocorreram durante a estiagem, e os menores no período de chuvas. Foi observado ainda que o município de Tufilândia mostrou-se mais diversificado quanto às espécies do fitoplâncton, além disso, apresentou também a maior riqueza específica e densidade total. Isso pode ser explicado pelas condições naturais que caracterizam os pontos localizados em Tufilândia. Nessa área, por exemplo, há menor turbulência da água (sem forte correnteza) e, consequentemente, áreas mais calmas.

A hipótese formulada de que existe influência das estações de chuvas e estiagem sobre a comunidade fitoplanctônica na região (variabilidade temporal da abundancia) foi confirmada pelo teste ANOSIM. Através dos resultados, foi possível observar uma diferença significativa entre a abundância do fitoplâncton nos períodos compreendidos neste estudo. A análise de agrupamento (CLUSTER) dos pontos amostrais, feita separadamente para os períodos de estiagem e chuvoso, foi baseada na ocorrência (presença e ausência) de espécies do fitoplâncton e permitiu identificar um agrupamento para os pontos localizados na cidade de Alto Alegre (P4 e P5) durante o período de chuvas e entre os pontos (P3 e P4) localizados na cidade de Tufilândia durante o período de seca. Esses agrupamentos, formados por pontos em diferentes localidades, podem refletir as condições in 
situ que promovem o desenvolvimento do fitoplâncton observadas nas áreas estudadas. Tais condições, como a baixa turbulência da água, observadas em ambos os locais de coleta, devem ter contribuído para uma similaridade entre os valores da abundância dos pontos amostrais agrupados, denotando, assim, uma relativa diferença entre os outros pontos quanto à presença das espécies.

O fitoplâncton do médio curso do rio Pindaré apresentou-se ricamente representado por uma grande variedade de espécies cuja ocorrência foi constante ao longo dos períodos estudados,sendo observadas em sua estrutura variações temporais na riqueza, diversidade e frequência de ocorrência. A grande diversidade foi marcada pela abundância de espécies de diatomáceas seguida das algas verdes. Foi possível observar a variação da comunidade em função da mudança no meio abiótico (estiagem e chuvas).

\section{Agradecimentos}

Os autores agradecem ao $\mathrm{CNPq}$, à Capes (Programa Capes Wageningen), à Prefeitura da cidade de Tufilândia pelo apoio logístico; ao senhor Tuló pelo auxílio nas expedições de campo, aos alunos Milena, Leonilde, Paulo e Ione pela ajuda nas atividades de coleta dos dados; ao DEOLI-LABOHIDRO e a todos que, de forma direta ou indireta, contribuíram para a realização e conclusão deste trabalho.

\section{Referências}

AHSAN, D. A. et al Plankton composition, abundance and diversity in Hilsa (Tenualosa Ilisha) migratory rivers of Bangladesh during spawning season. Dhaka University Journal of Biological Sciences v. 21, n. 2, p. 177-189, 2012.

ALMEIDA, I. C. S., et al. Comunidade fitoplanctônica do lago Cajari, Baixada Maranhense, no período de cheia. Boletim do Laboratório de Hidrobiologia v. 18 , n. 1, p. 1-9, 2005.

ALVIAL, I. E. et al. Estructura comunitaria de diatomeas presentes en los sedimentos superficiales de ocho lagos andinos de Chile central. Revista Chilena de Historia Natural v. 81, n. 1, p. 83-94, 2008

ARAÚJO, A et al. Fitoplâncton do rio Itapecuru, Maranhão, Brasil: uma contribuição ao conhecimento. Boletim do Laboratório de Hidrobiologia v. 11, n.1, p. 25-35, 1998.

AQUINO, E. P; et al. Fitoplâncton de uma lagoa de estabilização no nordeste do Brasil. Brazilian
Journal of Aquatic Science and Technology. v. 15, n. 1 , p. 71-77, 2011

BACIAS DO NORDESTE, 2000; Relatório estatístico hidroviário 1998, 1999 e 2000 Disponível em: http://www.transportes.gov.br/modal/hidroviario/ ESTATISTICA/BaciaPindare.htm. Acesso em: $18 / 10 / 2011$.

BARBIERI, R. et al. Plâncton, produção primária e alguns fatores físicos- químicos de dois lagos da Baixada Maranhense. Revista Brasileira de Biologia. 1989. v. 49, n. 2, p. 339-408, 1989.

BICUDO, C. E.; MENEZES, M. A. Gêneros de algas continentais do Brasil: chave para identificação e descrições. 2 ed. São Carlos, Rima. 502p, 2006.

BICUDO, C. E. M; BICUDO, T. M. R. Algas de água continentais brasileiras. Chave ilustrada para identificação de gêneros. Fundação Brasileira para o Desenvolvimento do Ensino da Ciência, São Paulo, 1970.

CARVALHO, M. C. Comunidade fitoplanctônica como instrumento de biomonitoramento de reservatórios no Estado de São Paulo. 2003. 83f. Tese (Doutorado) - Faculdade de Saúde Pública. Departamento de Saúde Ambiental. Universidade de São Paulo, São Paulo, 2003.

DELLAMANO-OLIVEIRA, M. J. et al. Os gêneros Staurastrum, Staurodesmus e Xanthidium (Desmidiaceae, Zygnemaphyceae) da Lagoa do Caçó, Estado do Maranhão, Nordeste do Brasil. Hoehnea. v. 35, n. 3, p. 333-350, 2008.

DELLAMANO-OLIVEIRA, M. J.; SENNA, P. A. C.; TANIGUCHI, G. M. Limnological characteristics and seasonal changes in density and diversity of the phytoplanktonic community at the Caçó pond, Maranhão State, Brazil. Brazilian Archives of Biology and Technology. v. 46, n. 4, p. 641-651, 2003.

LOPES, A. G. D. Estudo da comunidade fitoplanctônica como bioindicador de poluição em três reservatórios em série do Parque Estadual das Fontes do Ipiranga (PEFI), São Paulo, SP. 2007116 p Dissertação (Mestrado) - Faculdade de Saúde Pública. Departamento de Saúde Ambiental Universidade de São Paulo, São Paulo; 2007.

ESPINDOLA, E. G.; MATSUMURA-TUNDISI, T \& MORENO, I. D. Estrutura da comunidade fitoplanctônica da lagoa Albuquerque (Pantanal Matogrossense), Mato Grosso do Sul, Brasil Acta 
Limnologica Brasiliensia, v. 8, n. 1, 13-27, 1996.

ESTEVES, F. A. Fundamentos de limnologia, 3.ํe ed. Rio de Janeiro. Interciência. 826p, 2011.

FERRARI, F. et al. Eunotiaceae (Bacillariophyceae) em igarapés da Amazônia Central, Manaus e Presidente Figueiredo, Brasil. Acta Amazonica v. 37 , n. 1, p. 1-16, 2007

FRANCESCHINI, I.A.M, et al. Algas: uma abordagem filogenética, taxonômica e ecológica/ Iara Maria Franceschini... [et al] Porto Alegre: Artimed, 2010 332p, 2010.

GRANADO, D. C.; HENRY, R.; TUCCI, A. Influência da variação do nível hidrométrico na comunidade fitoplanctônica do Rio Paranapanema e de uma lagoa marginal na zona de desembocadura na Represa de Jurumirim (SP). Hoehnea. v. 36, n. 1, p. 113-129, 2009

HAMMER, Ø., D. Manual de Referência PAST: Paleontological Statistics Software Package for Education and Data Analysis. 2001. Disponível em: http://folk.uio.no/ohammer/past/. Acesso em 20/11/2011

HORNE, A.J. Limnology/ Alexander J. Horne, Charles R. Goldman-2nd Ed Library of Congress Catalogin-in-Publication Data 576p, 1994

LAUX, M.; TORGAN, L. C Diatomáceas com plastídeos no plâncton da foz dos rios do Delta do Jacuí, sul do Brasil: um complemento à taxonomia tradicional. IHERINGIA, Sér. Bot., Porto Alegre, v. 66, n. 1, p. 109 - 132, julho 2011.

MARANHÃO (Estado) Gerência de Planejamento e Desenvolvimento Econômico. Laboratório de Geoprocessamento - São Luis: Universidade Estadual do Maranhão (UEMA). São Luis, 44p. 2002

LORVERDE-OLIVEIRA, S. M et al. Fatores associados à distribuição espacial do fitoplancton em lagos de inundação (Pantanal Norte, Brasil). Oecologia Australis v. 16, n. 4, p. 770-781, 2012

MATTEUCCI, S. D.; COLMA, A. Metodologia para el estudio de la vegetación. Washington: Secretaria geral de la Organización de los Estados Americanos - Programa Regional de Desarrolo Científico y Tecnológico, 1982. 169 p, 1982.

MELO-MAGALHÃES, E. M.; et al. Diversidade e variação espaço-temporal do fitoplâncton no reservatório da Usina Hidroelétrica de Xingó.
Boletim de Estudos de Ciências do Mar. v. 11, p. 1-14, 2000.

MENEZES, V. C.; BUENO, N. C; RODRIGUES, L. C.; Spatial and temporal variation of the phytoplankton community in a section of the Iguaçu River, Paraná, Brazil. Brazilian Journal of Biology [online], v. 73, n. 2, p. 279-290, 2013.

MOSCHINI-CARLOS, V.; POMPÊO, MLM. Dinâmica do fitoplâncton de uma lagoa de duna (Parque Nacional dos Lençóis Maranhenses, MA, Brasil). Acta Limnologica Brasiliensia, v. 13, n. 2, p. 53-68, 2001.

MOSCHINI-CARLOS, V., et al. The planktonic community in tropical interdunal ponds (Lençóis Maranhenses National Park, Maranhão State, Brazil), Acta Limnologica Brasiliensia, v. 20, n. 2, p. 99-110, 2008

MÜLLER-MELCHERS, F.C.M.; FERRANDO, H. Tecnica para el estudio de las diatomeas. Boletim do Instituto Oceanográfico. v. 7, n. 1-2, p. 151-160, 1956.

ROUND, E.F. The taxonomic of the Chlorophyta, 2 Bot. Phycol., 6(2): 235-246. 1971

NOGUEIRA, N.M.C.. et al. Composition and temporal changes of phytoplankton community in Lake Quebra-Pote, MA, Brazil. Acta Limnologica Brasiliensia, v. 17, n. 4, p. 419-431, 2005.

OFFEM, B. O. et al. Plankton-Based Assessment of the trophic state of three tropical lakes. Journal of Environmental Protection, v. 2, n. 3, p. 304-315, 2011.

OLIVEIRA, M. A.; TORGAN, L. C.; RODRIGUES, S. C. Diatomáceas perifíticas dos arroios Sampaio e Sampainho, Rio Grande do Sul, Brasil. Acta Botanica Brasilica. V. 16, n. 2, p. 151-160, 2002

PEREIRA, A. C. A. Bioindicadores fitoplanctônicos da qualidade da água no estuário do rio Catu, Aquiraz, Ceará. 2007. 77f. Dissertação (Mestrado). Universidade Federal do Ceará, Fortaleza, 2007

POMPEO, M. L. M., et al. Heterogeneidade espacial do fitoplâncton no reservatório de Boa Esperança (Maranhão - Piauí, Brasil). Acta Limnologica Brasiliensia., vol. 10, n. 2, p 101-103, 1998

SILVA, V. L. Caracterização físico-química da água e sedimento do rio Pindaré, nos trechos

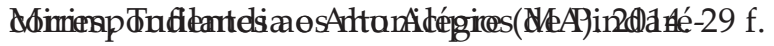


Monografia (Graduação). Universidade Federal do Maranhão, São Luis, 2014.

SOKAL R R \& ROBLI F I 1981. Biometry: the principles and practice $0 /$ statistics in biological research. San Francisco: W.H. Freeman, 1981. 859

UTERMÖHL, H. Zur Vervollkommnung der quantitativen Phytoplankton-methodic. Mitt. Internationale Ver. Theoretische und Angewandte Limnologie Germany, v. 9, p. 1-39, 1958.

TONETTA, D. PETRUCIO, M. M.; LAUDARESSILVA, R. Temporal variation in phytoplankton community in a freshwater coastal lake of southern Brazil. Acta Limnologica Brasiliensia [online]., v. 25, n. 1, p. 99-110, 2013 